\title{
Electrically-driven heterodyne detection of a multifrequency THz-wave with a photomixer
}

\author{
F. L. Constantin*a \\ ${ }^{a}$ Laboratoire PhLAM, CNRS UMR 8523, 59655 Villeneuve d'Ascq, France \\ *fl.constantin@univ-lille1.fr
}

\begin{abstract}
The spectral components of a multifrequency THz-wave may be characterized using the nonlinear electrical response of a low temperature grown GaAs photomixer. The heterodyne detection with the photomixer is exploited to down-convert a radiofrequency-modulated THz-wave to the microwave domain, by using a suitably tuned optical beat that acts as local oscillator. The photomixer is electrically driven with an alternative voltage oscillating at the modulation frequency and provides a phase-sensitive DC response signal indicating the demodulation of the THz-wave.
\end{abstract}

Keywords: frequency conversion, heterodyne detection, demodulation, low-temperature-grown GaAs, photomixer 


\section{INTRODUCTION}

At the crossroad of fundamental studies in molecular physics, optics and solid-state physics, the research with terahertz-waves enabled applications in astrophysics and atmospheric sciences, medical imaging, high-speed wireless communications, chemical sensing, and security screening ${ }^{1}$. The $\mathrm{THz}$ time-domain spectroscopy approach (THz-TDS) ${ }^{2}$ allowed broadband probing of the $\mathrm{THz}$ gap at the expense of a limited spectral resolution. The photomixing approach enabled optoelectronic generation of monochromatic THz-waves with broadband tuning ${ }^{3}$, and lead to compact, costeffective, room-temperature operated and low-power consuming $\mathrm{THz}$ photonic systems. The implementation of the optical frequency control provided narrow spectral linewidth, wide frequency tunability, high dynamic range and high signal-to-noise ratio. This contribution addresses the response to external electric fields of the LTG-GaAs photoconductor that was widely used as a photomixing platform. The nonlinearity of the response enables interplay between radiofrequency, terahertz and optical signals in the photoconductor leading to broadband frequency conversion. This phenomenon may be exploited in heterodyne detection, to down-convert to the microwave domain a radiofrequency-modulated $\mathrm{THz}$-wave by using a suitably tuned optical beat ${ }^{4}$. Here, a photomixer used for heterodyne detection is driven with an alternative voltage oscillating at the modulation frequency of the THz-wave in order to retrieve information about the modulation parameters of the THz electric field 5 .

\section{MULTIFREQUENCY PHOTOMIXING THEORY}

Two monochromatic laser beams, detuned by an angular frequency $\omega$ and carrying the same optical power $P$, are spatially superposed and enter at normal incidence in a LTG-GaAs photoconductor. The photogenerated carriers are driven by the electric field inside to the photoconductor, assumed to be oriented parallel with the surface of the photoconductor. The continuity equations allow to derive the expression of the current density :

$$
j(t)=\frac{2 e \eta P}{\hbar \bar{\top} A d}\left[\tau_{n}(E)_{v_{n}}(E)\left(1+\frac{\omega \tau_{n}(E) \sin (\omega t)+\cos (\omega t)}{1+\left(\omega \tau_{n}(E)\right)^{2}}\right)+\tau_{p}(E) v_{p}(E)\left(1+\frac{\omega \tau_{p}(E) \sin (\omega t)+\cos (\omega t)}{1+\left(\omega \tau_{p}(E)\right)^{2}}\right)\right]
$$

The lasers are focused on a photoconductive area $A$. The photons with nearly the same energy $\hbar \bar{\omega}$ are absorbed within a depth $d$, with a quantum efficiency $\eta$. The carrier lifetimes $\tau_{\mathrm{n}, \mathrm{p}}(E)$ increase 
nonlinearily with the applied electric field ${ }^{6}$. The linearized dependences utilized here are expressed with the contribution from carrier heating :

$$
\tau_{n, p}(E) \approx \tau_{0 n, 0 p}\left[1+3 \lambda e E /\left(2 k_{B} T\right)\right]
$$

in function of the Boltzmann constant $k_{B}$, the electron charge $e$, and the room temperature $T=300 \mathrm{~K}$. For the LTG-GaAs photoconductor, the low-field carrier lifetimes are $\tau_{0 n, 0 p}$ and the optical phonon mean free path is $\lambda$. The carrier drift velocities have nonlinear dependences on the electrical field ${ }^{7,8}$ that are expressed as :

$$
v_{n, p}(E)=v_{s n, s p} /\left[1+v_{s n, s p} /\left(\mu_{0 n, 0 p} E\right)\right]
$$

in function of the saturation velocities $\nu_{s n, s p}$ and the low-field mobilities $\mu_{0 n, 0 p}$ of the electrons and holes.

The total electric field in the photoconductor is assumed as the sum of the contributions from the bias and the radiofrequency voltages, that are applied to the photomixer electrodes, and from the Thévenin voltage induced by a THz-wave incident on the photomixer antenna :

$$
E(t)=E_{D C}+E_{R F} \cos \left(\omega_{R F} t+\phi_{R F}\right)+\sum_{n} E_{T H z, n} \cos \left(\left(\omega_{T H z}+n \omega_{R F}\right) t+\phi_{T H z, n}\right)
$$

The static electric field applied with the bias voltage is $E_{D C}$. The radiofrequency contribution is expressed with the amplitude $E_{R F}$, angular frequency $\omega_{A C}$, phase $\phi_{R F}$. The $\mathrm{THz}$ contribution is expressed for a THz-wave modulated at $\omega_{R F}$ displaying spectral components with amplitudes $E_{T H z, n}$ and phases $\phi_{T H z, n}$ at angular frequencies $\omega_{T H z}+n \omega_{R F}(\mathrm{n}$ integer). The electric fields in the photoconductor are expressed by assuming the parallel plate approximation : $E_{D C}=V_{D C} / r, E_{R F}=V_{R F} / r$, $E_{T H z}=\vec{E}_{T H z}^{i n c} \cdot \vec{l}_{\text {eff }} / r$, in function of the corresponding voltages, the electrode spacing $r$, the THz-wave electric field $\vec{E}_{T H z}^{i n c}$, and the effective vector length $\vec{l}_{e f f}$ of the antenna, respectively.

The electric field dependences $v_{n, p}(E)$ and $\tau_{n, p}(E)$ leads to a current density with spectral components at $d c$ and at the angular frequencies $\omega \pm n \omega_{T H z} \pm m \omega_{R F}$ where $m, n$ are integers. The dc current density, the amplitudes and the phases of the current density spectral components are calculated approximately by developing eq. (1) as a power series expansion in terms of the amplitudes of the oscillating fields at the Fourier frequencies by assuming a given bias electric field $E_{D C}$. The values 
used in these calculations for the parameters of the experimental setup and of the LTG-GaAs photoconductor are indicated in Table 1.

Let us discuss the predictions of this model for photomixing in the $\mathrm{THz}$ regime. First, the photomixer is operated with a radiofrequency voltage superposed on a dc voltage. The calculated values for the amplitudes and phases of the spectral components of the current density are displayed in Fig. 1(a). The current density displays equidistant spectral components. Three spectral components at $\omega$ and $\omega \pm \omega_{R F}$ have the same amplitude but slightly different phases, similarly to a $100 \%$ amplitude modulation signal. The phases and the amplitudes of the spectral components at $\omega+n \omega_{R F}$ and $\quad \omega-n \omega_{R F}$ are the same. The dependences in function of the amplitude of the radiofrequency electric field of the amplitudes and the phases of the spectral components are plotted in Fig. 1(b). For fields up to a few $10^{5} \mathrm{~V} / \mathrm{m}$, the increase of the amplitude for each sideband is associated with a stable phase.

Then, the unbiased photomixer is used for heterodyne detection of a THz-wave with three equidistant spectral components with the same amplitude and phase which are separated by a radiofrequency interval. The optical beat is detuned from the THz-wave frequency by a microwave interval. The results from numerical calculations for the spectral components of the current density are displayed in Fig. 2(a). The dc component is generated by rectification of the spectral components of the THz-wave. The spectral components in the radiofrequency domain are generated by beating the spectral components of the THz-wave on the nonlinear optically-driven photoconductance of the photomixer. The spectral components in the microwave domain are generated through downconversion of the THz-wave by heterodyne detection with the optical beat. These spectral components are equidistantly spaced by the radiofrequency interval around the microwave interval. Noteworthy, the current density displays also spectral components around the frequency of the optical beat and the THz-wave frequency that are equidistantly spaced by the radiofrequency interval.

While the rectification signal is not sensitive to the phases of the spectral components of the THzwave, the amplitudes of the radiofrequency components depend on their relative phases. In order to retrieve this phase information, the photomixer is then driven with a radiofrequency voltage with an adjustable phase. The amplitude of the spectral component at dc increases, as it shown on Fig. 2(b), 
and has a sinusoidal dependence on the adjustable phase. The number of the spectral components of the currant density in the radiofrequency, microwave and $\mathrm{THz}$ domain increase significantly that indicates efficient mixing in the photoconductor with the radiofrequency voltage.

\section{EXPERIMENTAL RESULTS}

The experimental setup (Fig. 3(a)) is based on a LTG-GaAs photomixer with a log-spiral antenna driven by the optical beat of two extended cavity laser diodes (DL1 and DL2) emitting at $820 \mathrm{~nm}$. The extremities of the antenna are connected to a microwave line addressed with a bias-T that allows coupling the signal from a radiofrequency synthesizer (RF) to the photomixer and to measure alternative components of the photomixing signal with a lock-in detection technique (Lock-in) or a microwave spectrum analyser (SA). The $\mathrm{THz}$ source is an active frequency multiplier driven by a microwave (MW) synthesizer. The output has $\mathrm{mW}$-level power in the 75-110 GHz frequency range. The synthesizers and the spectrum analyser are referenced against the signal of a GPS disciplined quartz oscillator.

The RF provides a signal at $15 \mathrm{MHz}$ that modulates the MW synthesizer. Upconversion yields a THz-wave with spectral components spaced by $15 \mathrm{MHz}$ around the carrier frequency at 102.072 GHz. The photomixer is used for heterodyne detection of the THz-wave. The spectrum of the detected signal using an unbiased photomixer is shown in Fig. 3(b). Then, a phase-delayed signal from the RF synthesizer with amplitude of $1.5 \mathrm{~V}$ is capacitively coupled to the electrodes of the photomixer. The signal at dc is recovered with the lock-in detection by using $100 \%$ amplitude modulation of the RF synthesizer at $1 \mathrm{kHz}$. The Fig. 3(c) presents the dependence of the detected signal in function of the relative phase between the THz-wave modulation signal and signal applied to the photomixer. The sinusoidal dependence indicates the demodulation of the photomixing signal at $15 \mathrm{MHz}$.

\section{CONCLUSION}

This contribution addresses the detection of THz-waves with a LTG-GaAs photomixer driven with an alternative electric field. The amplitudes and the phases of the photomixing signal are derived theoretically and investigated experimentally. Broadband frequency mixing, enabled by the nonlinear electrical response of the photoconductor driven with the optical beat of two continuous- 
wave lasers, is exploited for heterodyne detection and demodulation of a multifrequency THz-wave. This demonstrates the reversibility of the photomixing approach and enables integration of the functions of the optoelectronic detection and the electrically-driven demodulation in a single photonic circuit for $\mathrm{THz}$ signal processing. 


\section{REFERENCES}

[1] S.S. Dhillon, et al., "The 2017 terahertz science and technology roadmap,” J. Phys. D 50 (4), 043001 (2017).

[2] D. M. Mittleman, Sensing with THz Radiation, Springer-Verlag Berlin Heidelberg, 2003.

[3] E.R. Brown, K.A. McIntosh, K.B. Nichols, and C.L. Dennis, "Photomixing up to $3.8 \mathrm{THz}$ in low-temperature-grown GaAs,” Appl. Phys. Lett. 66 (3), 285-287 (1995).

[4] F.L. Constantin, "Phase-coherent heterodyne detection in the terahertz regime with a photomixer,” IEEE J. Quantum Electr. 47 (11), 1458-1462 (2011).

[5] F.L. Constantin, "Phase-coherent THz-wave demodulation with a photomixer," EPJ Web of Conferences 238, 12019 (2020).

[6] N. Zamdmer, and Q. Hu, "Increase in response time of low-temperature-grown GaAs photoconductive switches at high voltage bias,” Appl. Phys. Lett. 75 (15), 2313-2315 (1999).

[7] E.R. Brown, “A photoconductive model for superior GaAs THz photomixer,” Appl. Phys. Lett. 75 (6), 769-771 (1999).

[8] E.R. Brown, K.A. McIntosh, F.W. Smith, K.B. Nichols, M.J. Manfra, C.L. Dennis, and J.P. Mattia, "Milliwatt output levels and superquadratic bias dependence in a low-temperature-grown GaAs photomixer,” Appl. Phys. Lett. 64 (24), 3311-3313 (1994). 


\section{Figure captions}

Figure 1. Amplitudes and phases of the spectral components of the current density in a radiofrequency-driven photomixer driven with an optical beat at $\omega=2 \pi \times 1 \mathrm{THz}$. (a) Photomixing signal for $E_{D C}=5 \times 10^{5} \mathrm{~V} / \mathrm{m}, E_{R F}=8.35 \times 10^{5} \mathrm{~V} / \mathrm{m}, \omega_{R F}=2 \pi \times 1 \mathrm{GHz}, \phi_{R F}=0 \mathrm{rad}$. (b) Dependences on the amplitude of the radiofrequency electric field applied to the photomixer for $E_{D C}=5 \times 10^{5} \mathrm{~V} / \mathrm{m}$, $\omega_{R F}=2 \pi \times 1 \mathrm{GHz}, \phi_{R F}=0 \mathrm{rad}$.
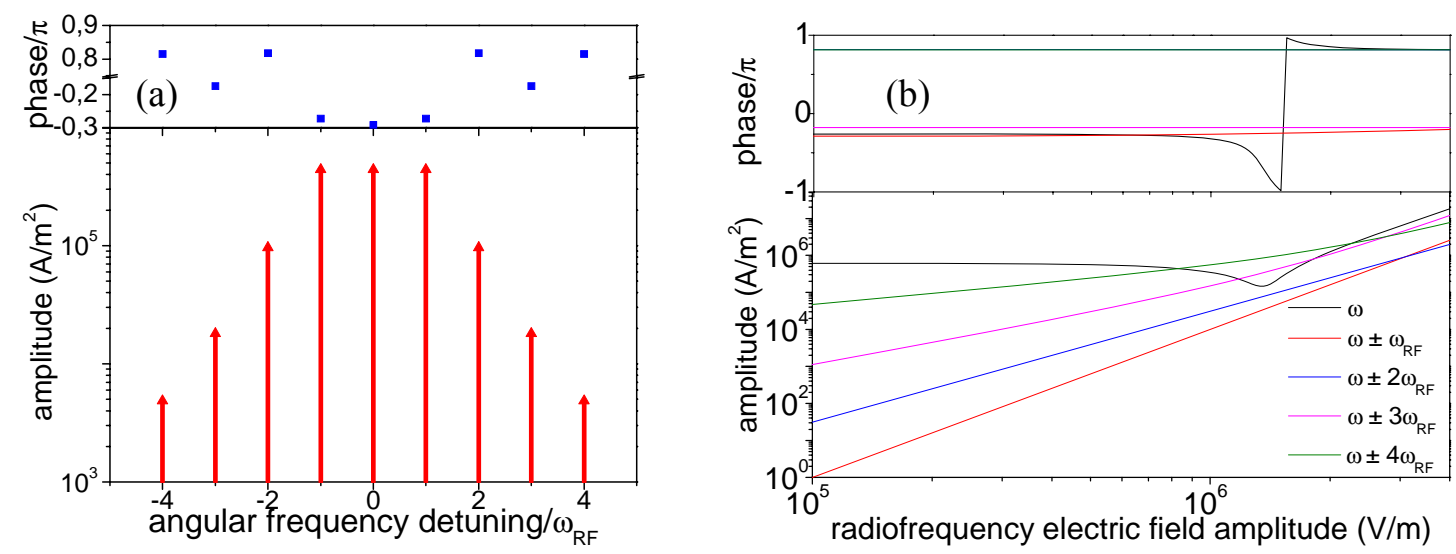

Figure 2. Heterodyne detection signals of a THz-wave with three spectral components at $\omega_{T H z}$, $\omega_{T H z}+\omega_{R F}, \omega_{T H z}+2 \omega_{R F}$. Amplitudes $E_{T H z, n}=10^{5} \mathrm{~V} / \mathrm{m}$ and phases $\phi_{T H z, n}=0 \mathrm{rad}$. Optical beat at $\omega=2 \pi \times 1$ THz. THz frequency at $\omega_{T H z}=\omega+\delta \omega=2 \pi \times 1.001 \mathrm{THz}$ and radiofrequency at $\omega_{\mathrm{RF}}=2 \pi \times 10 \mathrm{MHz}$. (a) Spectral components of the current density for an unbiased photomixer. $\quad$ (b) Spectral components of the current density for a photomixer operated using a radiofrequency electric field at $\omega_{\mathrm{RF}}=2 \pi \times 10$ $\mathrm{MHz}$ with amplitude $E_{R F}=10^{5} \mathrm{~V} / \mathrm{m}$ and phase $\phi_{R F}=0 \mathrm{rad}$.
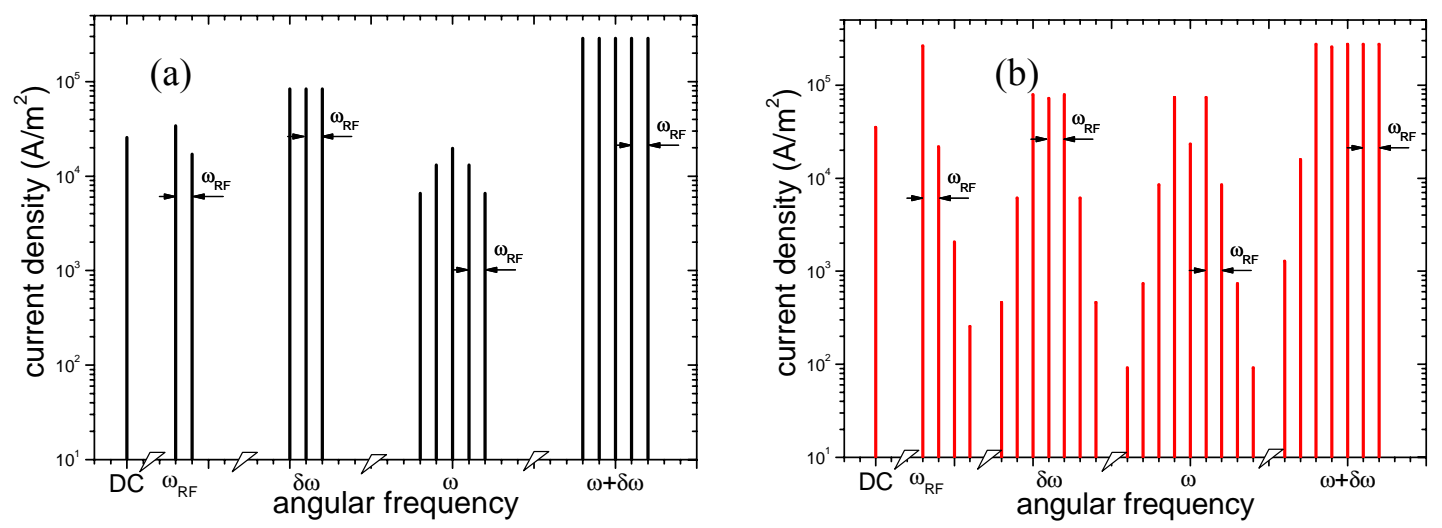
Figure 3. (a) Experimental setup. DL1, DL2 diode lasers, SA spectrum analyzer, THzFM frequency multiplier, RF and MW frequency synthesizers, Lock-in detection. The signal from RF is exploited to modulate the THz-wave and, upon application of an adjustable phase offset $\Phi$, to drive the photomixer electrodes. (b) Heterodyne detection signal of the THz-wave modulated at $15 \mathrm{MHz}$. Optical beat power $2 \times 14 \mathrm{~mW}$. RBW=1 MHz, sweep time $4 \mathrm{~ms}, 16$ video averages. (c) Phase dependence of the demodulated signal.
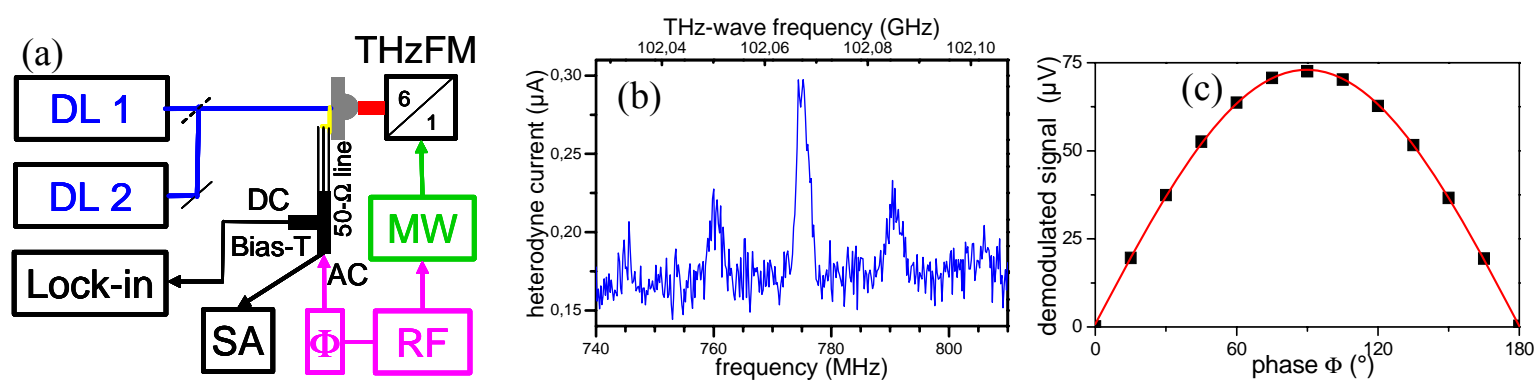

\section{Table captions}

Table 1. Characteristic parameters for the experimental setup and the photoconduction in the LTGGaAs layer.

\begin{tabular}{|l|l|l|l|}
\hline Photoconductor absorption depth $d$ & $2 \mu \mathrm{m}$ & Low-field hole lifetime $\tau_{0 p}$ & $0.4 \mathrm{ps}$ \\
\hline Photoconductor width $r$ & $2 \mu \mathrm{m}$ & Low-field electron mobility ${ }^{8} \mu_{0 n}$ & $400 \mathrm{~cm}^{2} / \mathrm{Vs}$ \\
\hline Laser power $P$ & $10 \mathrm{~mW}$ & Low-field hole mobility ${ }^{8} \mu_{0 p}$ & $100 \mathrm{~cm}^{2} / \mathrm{Vs}$ \\
\hline Photoconductive area $A$ & $12.56 \mu \mathrm{m}^{2}$ & Optical phonon mean free path $^{6} \lambda$ & $7 \mathrm{~nm}$ \\
\hline Laser frequency $v$ & $365.85 \mathrm{THz}$ & Dielectric constant $\varepsilon$ & 12.8 \\
\hline Electron saturation velocity ${ }^{7} v_{s n}$ & $40000 \mathrm{~m} / \mathrm{s}$ & Antenna resistance $R_{A}$ & $72 \Omega$ \\
\hline Hole saturation velocity ${ }^{7} v_{s p}$ & $10000 \mathrm{~m} / \mathrm{s}$ & Photomixer capacitance $C$ & $0.5 \mathrm{fF}$ \\
\hline Low-field electron lifetime $^{7} \tau_{0 n}$ & $0.1 \mathrm{ps}$ & & \\
\hline
\end{tabular}

